\title{
Nerve Involvement in Leprosy- Pathology, Differential Diagnosis and Principles of Management
}

\author{
J. M. H. PEARSON* AND W. F. ROSS \\ Medical Research Council Leprosy Project \\ and \\ All-Africa Leprosy and Rehabilitation Training Centre (ALERT) \\ P.O. Box 1005, Addis Ababa, Ethiopia
}

\begin{abstract}
Nerve damage in non-lepromatous leprosy and in "reversal" reactions is the result of an immune response to the presence of antigenic material derived from leprosy bacilli within nerves. This immune response damages nerves by intra-neural epithelioid cell formation, and by compression of Schwann cells due to inflammatory oedema. In lepromatous leprosy the presence of leprosy bacilli induces slow damage to both perineurium and Schwann cells. When Erythema Nodosum Leprosum (ENL) develops, associated neuritis is probably brought about by the presence of ENL lesions within the nerves.

In the absence of typical skin lesions, the nerve damage caused by leprosy can mimic other conditions. The differential diagnosis in such cases is outlined, and the principles of management of nerve damage due to leprosy are summarised.
\end{abstract}

\section{Some Definitions}

\section{NERVE INVOLVEMENT}

This is a general term, meaning anything from the presence of a leprosy bacillus in a nerve to the total destruction of the nerve.

\section{NERVE DAMAGE}

This implies that the presence of bacilli in a nerve or the tissue response to their presence, has caused structural alterations in the nerve. The mere presence of bacilli in a nerve need not be associated with nerve damage.

Nerve damage is recognized more readily histologically than functionally. Nerves that are clearly abnormal histologically can function without recognisable impairment. For instance, about $30 \%$ of the sensory fibres in a nerve must be destroyed before alterations of sensory acuity in the area it subserves can be detected.

\footnotetext{
* Requests for reprints should be addressed to J.M.H.P. at the National Institute for Medical Research, London, NW7 1 AA, England.
}

Received for publication 1 May, 1975. 
However, nerve damage is usually in terpreted in the clinical sense, that is, there are recognisable changes of nerve function. These can be detected by sensory testing (Pearson and Weddell, 1971), or by testing motor function by voluntary muscle power tests (VMT) (Goodwin, 1968). In some circumstances electromyographic techniques can be a sensitive guide to the progress or resolution of nerve damage (Sheskin et al., 1969).

\section{NEURITIS}

Literally "inflammation of nerve", it can be interpreted in the same sense as "nerve damage". However, in this paper, "neuritis" is defined as "pain and/or tenderness of nerve". The pain may be local or referred to the area of distribution of the nerve. Nerves which show neuritis usually also show recognisable impairment of function: but function can deteriorate in the absence of pain or tenderness.

\section{The Structure of Nerves}

The axons which are the actual conducting units of nerves are enclosed by the cytoplasm of Schwann cells. The larger axons are surrounded by a myelin sheath-in this case there is only one axon per Schwann cell. Smaller axons are unmyelinated, and there may be 10 or more in a single Schwann cell.

Apart from the terminal ramifications in skin and other tissues, the Schwann cells and their axons are wrapped in bundles, or fascicles: the tissue layer enclosing each bundle is the perineurium, a compact, multilayered structure which forms a relatively impermeable barrier between nerve and interstitial fluid. The larger fascicles contain one or more capillaries which run longitudinally and form a ladder pattern of connections within the fascicle. This capillary system is fed by arterioles and drained by venules which-penetrate the perineurium. Within the fascicle itself, however, it is uncommon to see any blood vessels save capillaries: and the direction of blood flow within an endoneurial capillary is unpredictable and probably variable (Lundborg, 1970).

The larger nerve trunks ("named nerves") are composed of many fascicles, which are bound together by the epineurium, a loose connective tissue sheath, which penetrates among the individual fascicles. The epineurium is vascular. Its longitudinal arterioles are fed at intervals by lateral connections from neighbouring arteries; but a normal nerve trunk can be deprived of its lateral blood supply for $15 \mathrm{~cm}$ or more and still maintain normal function (Bateman, 1962). Lymphatics are present in the epineurium but are not found within the individual fascicles.

\section{Initial Considerations}

\section{SITES OF NERVE INVOLVEMENT}

Nerves are chiefly involved in two zones, which are the same in all types of leprosy:

(a) Skin. The dermal nerves are microscopically involved in almost every case of leprosy, and some functional impairment can usually be detected by careful sensory testing. The subcutaneous nerves in the region of skin patches are also involved, and may be palpable. 
(b) Nerve trunks. These large nerves are chiefly affected and damaged at specific sites ("sites of predilection"); in most of these sites the nerves

(i) lie superficially, and so are liable to be cooler than average;

(ii) lie on bony structures, and so are exposed to damage due to repeated minor trauma.

\section{DIFFERENCES IN DIFFERENT TYPES OF LEPROSY}

Although nerves tend to be involved at the same sites in all types of leprosy, they are damaged more rapidly at the tuberculoid end of the spectrum. The tuberculoid skin lesion, by definition shows impairment of sensation, indicating that nerve damage is an early and intrinsic manifestation of this type of leprosy. By contrast, even fairly advanced lepromatous lesions may show only partial loss of sensation, and nerve trunk damage occurs late in the disease process. Nevertheless, patients with advanced lepromatous leprosy may have very extensive and severe loss of sensation, due chiefly to damage at the dermal nerve level. Patients with borderline leprosy are at a particular disadvantage: they have extensive nerve involvement, like lepromatous cases, and are liable to get severe and rapidly developing nerve damage, like tuberculoid cases.

\section{THE TIMING OF NERVE DAMAGE}

Nerves tend to be damaged only in two phases of the disease:

(a) In untreated leprosy. Nerve damage is part of the natural history of the disease; and patients are often suffering from nerve damage with secondary deformity when they are first diagnosed.

(b) During the course of reactions. Both type 1 reactions (reversal reactions, upgrading reactions) and type 2 reactions (Erythema Nodosum Leprosum, ENL) can affect nerves: and so nerve function can deteriorate during the course of treatment. However, in the absence of reactions, patients receiving adequate anti-leprosy treatment usually do not develop further nerve damage, and indeed nerve function can improve (though the deformity secondary to established nerve damage may worsen).

\section{IMMUNOLOGICAL CONSIDERATIONS}

(a) Peripheral nerves are well protected structures. They are unaffected by pyogenic infections around them, and are never the site of tuberculomas. Mycobacterium leprae and some viruses are the only pathogens which regularly enter nerves.

(b) This protection is related to the structure of the nerve. In particular, the multilayered perineurium is structually well suited to a barrier function; and the endothelial cells of the intraneural capillaries have tight junctions, rendering them less permeable than blood vessels elsewhere.

(c) The relative chemical isolation of nerve fibres is also an immunological isolation. Peripheral nervous tissue can be described as an "immunological backwater".

(d) Schwann cells have a long life span; once within these cells leprosy bacilli may not be re-exposed $t \bar{Q}$ he extracellular environment for years. Soluble antigens may leak out, but even so the infection in this sheltered zone can escape recognition by the host defences for prolonged periods of time. This situation is in contrast to that of extraneural bacilli phagocytosed by 
histiocytes; the life span of these cells is a matter of weeks only. Soluble antigens may be exposed at any time: but when the host cell dies whole bacilli are re-exposed to the extracellular environment.

\section{The Entry of Bacilli Into Nerves}

There are three possible ways in which the bacilli can enter peripheral nerves:

(1) By phagocytosis by Schwann cells in the upper layers of the dermis. These cells show "turnover" and can be actively phagocytic. Once engulfed, the bacilli could travel by contiguity from Schwann cell to Schwann cell along the nerve, or possibly within the axon. There are a number of objections to this hypothesis:

(a) There is little evidenee that bacilli commonly lie freely in the dermis, (except in the rather avascular subepidermal zone in tuberculoid and borderline cases).

(b) Histologically the nerves in the middle and deeper layer of the dermis are of ten involved to a greater extent than the terminal nerve fibres.

(c) Bacilli are very seldom seen in axons (Boddingius, 1974).

(2) By penetration of the perineurium. However, this membrane presents a formidable barrier; in leprosy, when inflammatory cells are penetrating the perineurial layer, the nerve is usually bacillated: the invasion is the consequence of the presence of bacilli rather than the cause of their entry.

(3) Via the endoneurial blood vessels. This is the likeliest hypothesis, for there is good evidence that even tuberculoid leprosy is a systemic disease (Pearson et al., 1970; Karat et al., 1971); and bacteraemia is a normal finding in patients with untreated lepromatous leprosy (Drutz et al., 1972). Also in early nerve involvement the bacilli tend to lie close to endoneurial blood vessels.

Much, however, is still unknown. For instance, it is not yet known whether bacilli are carried into nerves within macrophages or, lying free, adhere to the endoneurial capillaries and are thus trapped. But once they have entered a nerve, their ultimate phagocytosis by Schwann cells is probably inevitable.

\section{The Localization of The Intraneural Infection}

The sites of the body where nerves are most readily colonized and damaged are probably cooler than the average body temperature (Sabin et al., 1974), and it is possible that bacilli multiply more readily in these sites. Nevertheless the focal involvement of nerve trunks appears to be more than can be accounted for in this way. Of other factors that could be involved in this localization, the most likely is trauma. When a nerve is exposed to constant minor trauma, it is likely to be mildly inflamed, and the consequent increased capillary stickiness at these sites may make bacilli or monocytes more likely to adhere and penetrate the nerve. The sites of predilection of nerves may therefore have the nature of traps for circulating bacilli. Nevertheless other mycobacteria do not enter nerves, and there is as yet no satisfactory explanation for this unique property of Mycobacterium leprae.

\section{The Evolution of the Intraneural Infection}

In the initial stages of the infection, Schwann cells are colonized by $M y c o$. leprae, but seem to be little affected by their presence. However, af ter a period the Schwann cell may be destroyed, possibly by the bulk of bacilli it contains, or 
by interference with its metabolic processes. The bacilli are released, and may be taken up by neighbouring Schwann cells or possibly by in traneural histocytes which could transport them via the endoneurial blood vessels along the nerve. Thus the intraneural infection can progress slowly along the nerve. In established tuberculoid leprosy, where bacilli are markedly more concentrated in nerves than elsewhere, such intraneural spread is probably the major route of dissemination of the infection.

\section{The Host Response to the Infection}

\section{(1) TUBERCULOID LEPROSY}

Nerves are "immunological backwaters"; but, as bacilli multiply within a nerve, soluble antigen leaks out of the Schwann cells into the endoneurial blood vessels and through the perineurium. Thus the infection is recognized, and the host defences mount an attack. The nerve is invaded by inflammatory cells, chiefly through the perineurial layers, possibly because the circulation in the endoneurial capillaries disperses the antigen before a critical "recognition level" is attained. The result is the formation of an epithelioid cell granuloma within the nerve; the Schwann cells and the axons they contain are destroyed in the process (see Fig. $1)$.

(a)

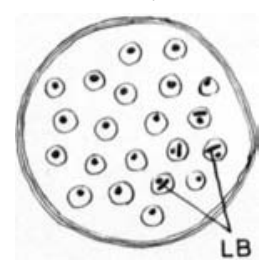

(b)

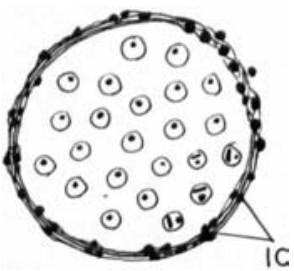

(c)

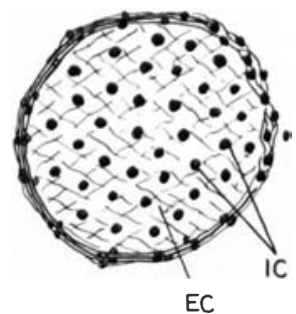

Fig. 1. The process of nerve damage in tuberculoid leprosy. (a) A nerve fascicle with a few leprosy bacilli (LB) lying within Schwann cells. (b) Inflammatory cells (IC) cluster round the perineurium and begin to infiltrate its layers. (c) Inflammatory cells (IC) have entered the nerve, with consequent epithelioid cell (EC) formation.

In tuberculoid leprosy, bacilli spread slowly along the dermal nerves: the bacillary concentration must reach a "critical level" before the infection is recognized, and attacked. If all the bacilli were destroyed, this would be a true, self-limiting infection. But normally some bacilli survive, and so the tuberculoid skin lesion slowly extends. The process may be visualized as a wave of bacillation spreading slowly through the dermal nerves, pursued but never quite overtaken by the inflammatory response. The infection is largely confined to nerves; once they are all destroyed, the bacilli are destroyed with them, and resolution by fibrosis occurs, forming the "clear centre" of the tuberculoid lesion.

\section{(2) BORDERLINE LEPROSY}

In this type of disease the nerves are attacked in the same way. But the host cells are less highly sensitized to the infection, and higher bacillary concentrations are required to elicit an inflammatory response. Bacilli are therefore present in 
considerable numbers in Schwann cells, and sometimes can be seen in the perineurium. Also the process is more focal and less acute and destructive. The morphology of the nerves is better preserved, and characteristically there are zones of epithelioid cells adjacent to areas where the Schwann cells, though bacillated, are well preserved. Not infrequently the epithelioid cell foci lie next to the perineurium (and may indeed form within the layers of the perineurium (Pearson and Weddell, 1975)). In such cases the appearance is of strands of surviving Schwann cells lying in an annular zone of inflammatory tissue (see Fig. 2).

(a)

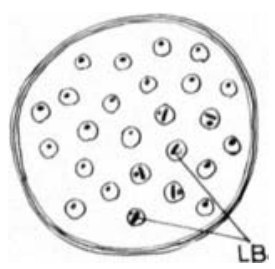

(b)

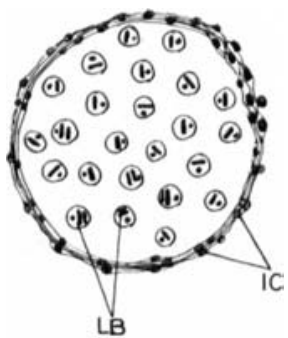

(c)

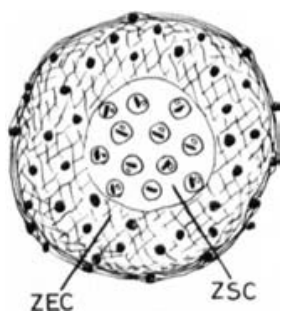

Fig. 2. The process of nerve damage in borderline leprosy. (a) A nerve fascicle with a few leprosy bacilli (LB) lying within Schwann cells. (b) The leprosy bacilli (LB) are considerably increased in number, and inflammatory cells (IC) are beginning to infiltrate the perineurium. (c) Inflammatory cells have entered the nerve, giving rise to a zone of epithelioid cells (ZEC). There is a zone of surviving bacillated Schwann cells (ZSC).

\section{(3) LEPROMATOUS LEPROSY}

In this type of disease the host preserves little or no capacity to destroy the invading mycobacteria-consequently they multiply unchecked, and in almost any situation. Thus, in nerves the bacillary concentration in perineurial cells is at least as great as in the Schwann cells, and often greater. Both sites are damaged, and the perineurial changes may be the more important in the causation of nerve damage in lepromatous leprosy.

(a)

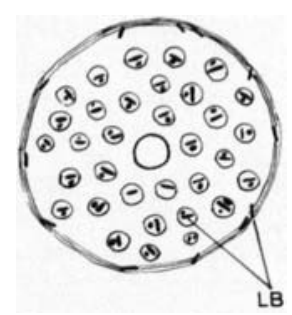

(b)

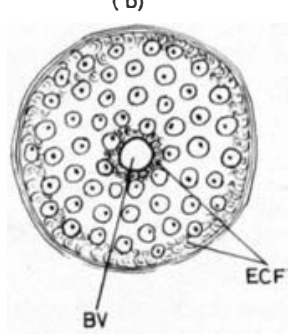

(c)

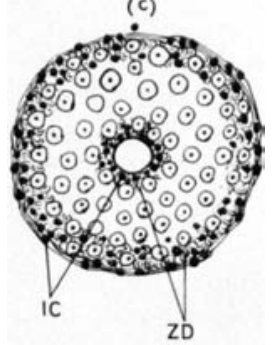

Fig. 3. The process of nerve damage in lepromatous leprosy. (a) Leprosy bacilli (LB) are seen in the Schwann cells and also in perineurial cells of a nerve fascicle. (b) Extracellular fluid components (ECF) leak through the perineurium and intraneural blood vessels (BV) into the nerve. (c) Inflammatory cells (IC) enter the nerve giving rise to zones of damage (ZD) round the blood vessel and near the perineurium.

NOTE. Leprosy bacilli have been omitted from diagrams (b) and (c). 
The perineurium acts as a barrier between Schwann cells and the extracellula! fluid; it plays a major part in stabilizing the intraneural environment.. In lepromatous leprosy, bacillary multiplication within the perineurial cells appears to initiate the breakdown of this barrier; the process can be visualized as the formation of a series of "micropunctures" in the perineurium. There are two consequences of this "perineurial incompetence" (Fig. 3):

(a) Extracellular fluid components can enter the nerve; this can impair the function of Schwann cells. Such impairment appears, on occasions at least, to be reversible, and may account for the very rapid improvement of nerve function that sometimes occurs in the early months of treatment of lepromatous leprosy.

(b) The nerve appears also to be more vulnerable to the entry through the perineurial layers of inflammatory cells. Thus, in the nerve zone immediately adjacent to the perineurium there can be seen a "zone of damage" where inflammatory cells are present in higher concentration than in the rest of the nerve, and where the Schwann cells have sustained more damage. Similar zones of damage are to be seen around the intraneural blood vessels, whose endothelial cells often contain large numbers of bacilli, and which have been shown in experimental leprosy to be abnormally permeable (Boddingius et al., 1972).

Experimental damage to the perineurium has been shown in normal animal nerve to elicit a cellular response which has the effect of repairing the damage (Morris et al., 1972). In patients with lepromatous leprosy this process can be most clearly seen in the dermal nerves; the end result is the transformation of the perineurium into a wide "perineurial zone" made up of multiple strands of perineurium, which take on a thick fleshy appearance, and whose cytoplasm contains many bacilli. The layers of perineurium are infiltrated with inflammatory cells, chiefly histiocytes and plasma cells; and on occasion the layers are solidly filled with cells, giving an onion peel appearance. There are usually a higher concentration of bacilli in the perineurial zone than in the surviving Schwann cells.

The mechanism which causes this appearance is by no means certain. In the experimental situation of Morris et al., 1972 it was clear that Schwann cells took part in the process of perineurial repair; they formed circumferential elongations around groups of other Schwann cells, thus giving rise to a "pseudoperineurium", which in time became organized till it was indistinguishable from normal perineurium. Thus, under their experimental conditions Schwann cells could metamorphose to take on the form and function of perineurial cells.

It is likely that a similar process occurs in leprosy, in an attempt to repair the perineurial damage. The process is shown diagrammatically in Fig. 4. Schwann cells in the region of perineurial micropunctures divest themselves of their axons, extend lateral processes and apply themselves as "patches" to the damaged area. However, the Schwann cells themselves will probably be or become bacillated, and the patching process, though unlikely to be functionally effective, will be liable to continue, thus giving rise to the multilayered perineurial appearance. As this process continues, the number of Schwann cells will slowly decrease, and so the nerve will slowly be damaged, and finally destroyed.

If this hypothesis is correct, it accounts for the fact that in lepromatous leprosy there is of ten remarkable preservation of motor nerve function even in advanced cases with severe dermal nerve damage and sensory deficit. If the process of 
(a)

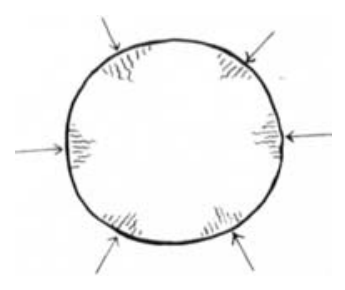

(c)

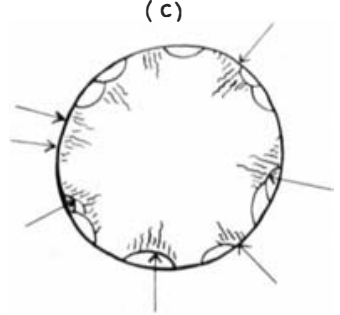

(b)

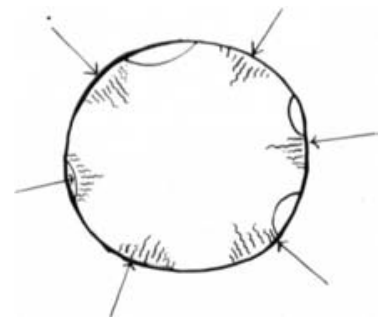

(d)

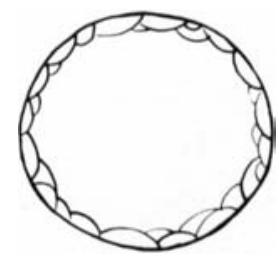

(e)

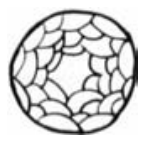

Fig. 4. The possible sequence of events which causes the multilayered appearance of the perineurium in lepromatous leprosy. $\rightarrow$, site of "micropuncture"; SSS, zone into which extracellular fluid components leak. (a), (b), (c), and (d) show the evolution of this appearance, (e) shows a small nerve almost destroyed by a depth of multilayering which leaves a larger nerve (d) largely intact.

damage involves the slow destruction of Schwann cells and their incorporation into the perineurial layers, it is likely to extend gradually into nerves, and should spread at much the same rate regardless of the diameter of the nerve. Thus a small nerve can be fully destroyed at a time when a large nerve is only affected at its periphery, and still retains the majority of its functional capacity (Fig. 4(e)). In other words, leprosy does not have a predilection for sensory nerves, but in lepromatous leprosy it takes much longer to destroy the larger nerves.

Final proof of the part played by Schwann cell metamorphosis in the development of the nerve damage in lepromatous leprosy will require much experimental work; but it is clear that perineurial damage, however caused, and whatever its consequences, plays a major part in the causation of nerve damage in lepromatous leprosy.

\section{(4) TYPE 1 REACTIONS (REVERSAL OR UPGRADING REACTIONS)}

These reactions occur most characteristically in borderline leprosy, and tend to develop in the early months of treatment, though much the same process can occur in untreated cases. They are associated with an increase of the immune response of the host cells to certain antigens of Myco. leprae, and are clinically characterized by the development of increased oedema and erythema of skin lesions. Nerves are commonly involved, and can be rapidly damaged, sometimes with little or no pain or tenderness.

Histologically the nerves in borderline leprosy can be considered as consisting of strands of surviving Schwann cells in tubes of rather rigid inflammatory tissue (Fig. 5). They are clearly very vulnerable to pressure; and in the early stages of 
(a)

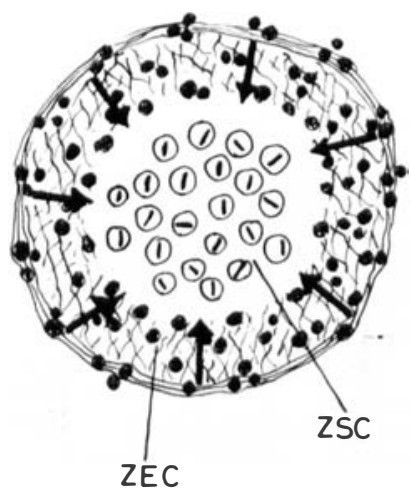

(b)

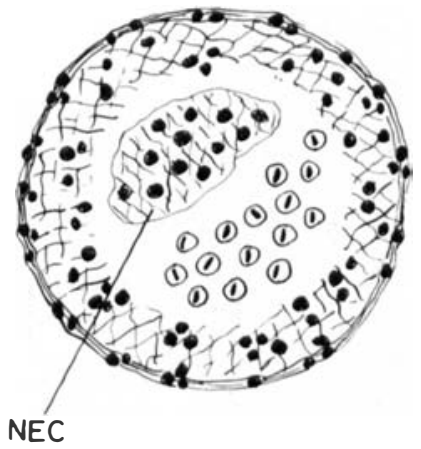

Fig. 5. The process of nerve damage in "reversal" reaction. (a) Oedema of the epithelioid cell zone (ZEC) causes compression of the surviving zone of Schwann cells (ZSC). (b) Shows formation of new epithelioid cells (NEC) in the zone of surviving Schwann cells.

Type 1 reaction the first and invariable histological feature is increased oedema of the granuloma. Thus the surviving strands of Schwann cells are liable to be suddenly compressed, and nerves can lose their function extremely rapidly for this reason. If the pressure is relieved before demyelination occurs, function can recover within a few days; if the axons have been demyelinated, recovery will require longer, probably several weeks. If the pressure is sufficient and prolonged enough to cause actual interruption of the axons, recovery must be delayed till regeneration occurs, usually several months.

Compression, however, is not the only way in which these nerves can sustain damage. The increased sensitivity of the host cells may be sufficient to cause further epithelioid cell formation within the nerve if the concentration of bacillary antigen in the surviving Schwann cells is sufficient to trigger the response. In these circumstances the same response will occur as is seen in the untreated disease; inflammation within the nerve, with consequent destruction of the Schwann cells and their axons. Permanent and irreversible nerve damage is liable to ensue.

\section{(5) ERYTHEMA NODOSUM LEPROSUM (ENL)}

This reaction appears to be the consequence of the formation of antibody/ antigen complexes (immune complexes), which are toxic and cause tissue damage. They can form wherever there is bacillary antigen present, and though the name implies that it is a skin condition, lesions can cause neuritis, orchitis, lymphadenitis, and even polyarthritis, and renal involvement (presumably associated with the deposition of circulating immune complexes).

At the clinical level, the chief difference between the neuritis associated with ENL and that occurring in Type 1 reactions is that nerve damage occurs much more slowly in the former. In reversal reactions, there can be rapid nerve destruction with little neuritis. In ENL nerves can be painful and tender for prolonged periods, and still show only mild loss of function. Thus the treatment 
of neuritis in ENL is much less urgently required than that of nerve involvement in Type 1 reactions, even though the symptoms may be more severe.

The pathological changes associated with neuritis in patients with lepromatous leprosy are less fully understood than are those of Type 1 reactions. ENL tends to occur in patients who have been treated for some months or years; in these cases the processes of resolution of the dermal nerve involvement due to uncomplicated lepromatous leprosy have been largely completed. Indeed biopsies in such cases not uncommonly show well preserved and apparently protected and spared small dermal nerves lying close to or within foci of polymorphs in skin ENL lesions. The fact that ENL nodules tend to be very tender also implies that the nerves are functioning efficiently; the pain is probably chemically induced by the release of active substances such as polypeptides from damaged cells in the vicinity of the nerves.

In nerve trunks, biopsies taken when the nerve is painful and tender will, on occasion, show the presence of ENL lesions of the same sort as are seen in skin. But this is by no means invariable; not uncommonly such biopsies show surprisingly little abnormality, merely scattered inflammatory cells among the Schwann cells. The reason for this is uncertain: possibly the biopsy was taken too late, after the lesion had resolved, or possibly there was a localized ENL lesion elsewhere in the nerve. Or it is possible that when ENL affects nerves its manifestation can differ from that seen in skin.

If the neuritis seen in patients with lepromatous leprosy, and often accompanied by ENL of the skin, is indeed a different process from that seen in

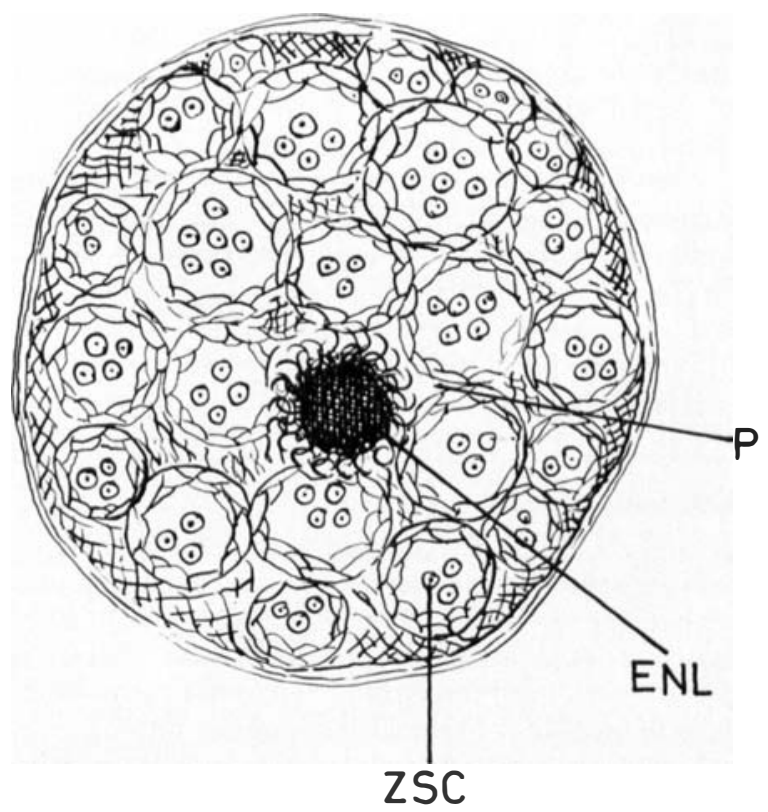

Fig. 6. The process of nerve damage in ENL. There is an ENL focus (ENL) within a nerve trunk. It lies between zones of surviving Schwann cells (ZSC) among the layers of perineurium (P). 
the skin, this could account for the slowness with which nerve damage occurs in these cases. But even if actual ENL nodules form within nerve trunks, as is sometimes demonstrated, there is a possible explanation for the unexpectedly slight damage they usually cause. The nerves in such patients are usually severely damaged, the process of perineurial "proliferation" is far advanced, and the whole structure of the nerves distorted by the ingress of inflammatory cells. Indeed it is of ten difficult to recognize that such biopsies are composed of neural tissue. Moreover, as in untreated lepromatous leprosy, there is a tendency for the bacillary concentration to be greater in the perineurial zone. Thus it is possible that ENL lesions, which tend to develop in areas of higher bacillary concentration, will occur chiefly in perineurial areas, and thus be less liable to damage the surviving Schwann cells (Fig. 6).

\section{Differential Diagnosis of Nerve Involvement}

\section{(1) LEPROSY AND OTHER DISEASES}

The salient facts to bear in mind so far as the neurological changes in leprosy are concerned are:-

(a) Localized irregular nerve enlargement is almost always due to leprosy.

(b) Muscle weakness is always due to lower motor neurone damage, and almost never attacks proximal limb muscles, limb girdle muscles, or the trunk musculature.

(c) In early cases of leprosy sensory loss shows a mixture of "named nerve" distribution together with localized areas of impaired sensation due to dermal nerve damage in skin lesions.

(d) A "glove and stocking" pattern of sensory loss can be found, usually in more advanced cases. But careful sensory testing will almost always reveal small islands of preserved sensation on the feet and hands. Also the tendon reflexes are preserved in these cases, and are often brisker than usual.

(e) Position sense is almost always preserved.

(f) The central nervous system is never damaged by leprosy.

Diseases most commonly confused with leprosy include:-

(a) Those associated with spinal cord abnormalities, such as syringomyelia, amyotrophic lateral sclerosis, and motor neurone disease.

(b) Those associated with peripheral nerve lesions.

(i) Damage by pressure, such as spinal root compression, the carpal tunnel syndrome and Bell's palsy.

(ii) Polyneuritis, which may be:-

Hereditary - Hypertrophic interstitial neuropathy, peroneal muscular atrophy.

Metabolic - Diabetes mellitus, porphyria, amyloidosis.

Deficiency - Vitamin B1 or B12 especially, precipitated by malabsorption, alcoholism, or other malnutrition.

Toxic - Lead, mercury, arsenic.

Industrial and therapeutic chemicals.

Diphtheria and other infections.

Malignant - Particularly with carcinoma of the bronchus. 
(c) Those associated with muscle disease, including the hereditary myopathies and myositis.

(d) Those associated with "trophic" changes, including diabetes mellitus, tabes dorsalis and congenital indifference to pain.

In most cases awareness of these possibilities, together with a full history and clinical examination, are sufficient to make the correct diagnosis.

\section{(2) DIFFERENT TYPES OF NERVE DAMAGE IN LEPROSY}

There is seldom a problem of differential diagnosis in patients newly presenting with untreated leprosy and nerve involvement. Once the type of leprosy is know the process occurring in the nerves can be considered reasonably certain. It is unusual in these cases for very rapidly progressive nerve damage to be occurring, and in general the initiation of anti-leprosy treatment will control the progress of nerve damage. The more common problem is that of patients who develop nerve involvement during the course of treatment. In such cases also, however, the key to diagnosis is the classification of the disease.

In tuberculoid leprosy, borderline leprosy and Type 1 reactions the mechanism of nerve damage is almost identical, differences being related to the phase of the disease and to the severity and suddenness of onset. Even untreated cases can develop a fairly sudden increase of oedema and erythema of their skin lesions together with neuritis; and clinically the distinction between active leprosy, very active leprosy, and reaction probably cannot be defined. In the times before effective treatment was known, such reactions tended to leave the patient closer to the lepromatous end of the spectrum, and have therefore been called "downgrading" reactions (Ridley, 1969). However, it seems likely that they are brought about by the same mechanism as in "reversal" reactions, namely, an increased immune response to bacillary antigens; and these downgrading reactions are probably best considered to be host responses to antigens which are not concerned with resistance to the infection.

Thus, nerve involvement, whether detected by the patients' subjective symptoms, by the presence of neuritis, or by tests to assess nerve function, represents the same process in non-lepromatous leprosy, namely the damage of nerve tissue by the host response to mycobacterial antigens contained in the nerve. It is an unwanted part of the immune response to the infection. Any patient with non-lepromatous leprosy is liable to develop such nerve involvement, either before treatment starts or during treatment so long as there is bacillary antigen remaining in the nerves.

In lepromatous leprosy under treatment, nerve damage, with or without neuritis, is commonly associated with ENL lesions in the skin; and whether such lesions are present or not, the nerve involvement probably represents the same process, namely immune complex formation occurring within the nerves. Occasionally, however, patients with subpolar lepromatous leprosy can develop severe nerve damage associated with epithelioid cell formation within the nerve, although there may be no sign of Type 1 reaction in the skin.

\section{Principles of Management of Nerve Involvement}

Nerve damage occurring in the course of the untreated disease may be irreversible, but it is usually arrested by the initiation of anti-leprosy treatment. If however, the disease is unusually active and nerves are being acutely damaged, 
management should be on the principles outlined below for the treatment of nerve involvement in reactions.

When nerves are being damaged in the course of treatment, it is normally because a reaction is present. Such reactions can occur in nerves without affecting the skin; this is presumably related to the different concentrations of bacillary antigen in different sites, and particularly to the way in which bacilli can survive for prolonged periods in nerves, even in non-lepromatous cases.

\section{(1) NERVE DAMAGE IN "REVERSAL" (TYPE 1) REACTIONS}

In non-lepromatous leprosy nerve damage is caused by the immune response of the host. The appropriate therapy therefore is immunosuppression, and the usual drugs those of the corticosteroid group. The possibility of very rapid and severe nerve damage in such cases should encourage the use of steroids. They relieve inflammatory oedema, and so lessen nerve compression; and by their immunosuppression prevent further epithelioid cell formation within nerves.

Steroid therapy must be in adequate dosage to control the reaction, and continued until the conditions which precipitated the reaction are no longer present; this means, until the concentration of bacillary antigen in the nerves is reduced to a level that no longer elicits an immune response. In most cases, the period required is a few months, so the dangers of ultra long term treatment with steroids are unlikely to occur. Dosage levels higher than prednisolone $30-40 \mathrm{mg}$ daily are seldom required, and can usually be reduced to about $20 \mathrm{mg}$ daily after 46 weeks. It is important to continue anti-leprosy treatment, and there is little evidence that the drug or dosage employed influences the end result.

\section{(2) NER VE DAMAGE IN ENL (TYPE 2) REACTIONS}

In lepromatous leprosy neuritis is not uncommon, and may or may not be associated with the presence of ENL lesions in the skin. However, loss of function is much slower to develop than in reversal reactions. Nerves can be tender and painful and yet function well; and patients can show nerve tenderness for months and still develop little or no deterioration of function. This consideration is the major reason for the different management of neuritis in lepromatous leprosy, in which the corticosteroids should not be used in the first instance, but reserved for patients showing nerve damage as well as neuritis. When nerve function is not deteriorating the "standard" drugs for the treatment of ENL should be employed; these include chloroquin, stibophen (fouadin), analgesics, and, in more severe cases, clofazimine and thalidomide. Local treatment, particularly splinting, is often helpful in relieving pain, as are perineurial injections of local anaesthetic and steroids. The place of surgical treatment in the management of nerve damage has yet to be determined.

When treating patients with ENL and neuritis, the normal pattern of the condition should be borne in mind. ENL is usually episodic; an attack can last for a week or two, but may not recur for weeks or months. Corticosteroids, if indicated, should be prescribed on this principle; fairly high dosages (say $30 \mathrm{mg}$ prednisolone daily) are likely to be required only for a few days, and the dosage should be rapidly reduced, the whole course lasting no longer than 2-3 weeks, and repeated as necessary if severe neuritis recurs. The occasional patient who develops prolonged uninterrupted neuritis associated with nerve damage or severe ENL may require continuous steroid treatment; but the requirement can usually be much reduced by the concomitant use of clofazimine and/or thalidomide. 
Anti-leprosy treatment must be continued, and there is little evidence that the drug or dosage employed affects the result. (Clofazimine is of value because of its anti-inflammatory activity: its anti-leprosy action is an incidental benefit.)

\section{(3) ESTABLISHED NERVE DAMAGE}

Although much nerve damage in leprosy is in practice unpreventable, it need not lead on inevitably to the deformities which are the popular hallmark of the "leper". Deformity of the hands and feet in leprosy is associated with tissue injuries (in anaesthetic limbs) and muscle imbalance due to motor nerve damage and consequent weakness of certain muscle groups. But the stiffness of joints is due to failure to put them through full range movements regularly. Simple health education, aiming to teach patients the hazards of anaesthesia and how to avoid them; skin care, and particularly, the early treatment of minor injuries; and simple exercises to be performed for a few minutes daily, can largely prevent the development of deformities even if there is permanent nerve damage.

\section{Acknowledgements}

We are most grateful to Miss Jane Neville, who drew the diagrams, and to Prof. A. G. M. Weddell, who shared in most of the histopathological studies summarised here. He and many other colleagues have stimulated us in discussions and advised in the preparation of this paper.

\section{References}

Bateman, J. E. (1962). Trauma to Nerves in Limbs. W. B. Saunders Co., Philadelphia and London.

Boddingius, J. (1974). The occurrence of Mycobacterium leprae within axons of peripheral nerves. Acta neuropath. 27, 257.

Boddingius, J., Rees, R. J. W. and Weddell, A. G. M., (1972). Defects in the "blood-nerve barrier" in mice with leprosy neuropathy. Nature, New Biol. 237, 190.

Drutz, D. J., Chen, T. S. H. and Lu, W. H. (1972). The continuous bacteraemia of lepromatous leprosy. New. Engl. J. Med. 287, 159.

Goodwin, C. S. (1968). The use of the voluntary muscle test in leprosy neuritis. Lepr. Rev. 39, 209.

Karat, A. B. A., Job, C. K. and Rao, P. S. S. (1971). Liver in leprosy-histological and biochemical findings. Br. med. J. 1, 307.

Lundborg, G. (1970). Ischaemic nerve injury: experimental studies on intraneural microvascular pathophysiology and nerve function in a limb subjected to temporary circulatory arrest. Scand. J. Plastic Reconstructive Surgery, Supplementum 6.

Morris, J. H., Hudson, A. R. and Weddell, G. (1972). A study of degeneration and regeneration in the divided rat sciatic nerve based on electron microscopy. 4. Changes in fascicular microtopography, perineurium, and endoneurial fibroblasts. Z. Zellforsch. mikrosk. Anat. $124,165$.

Pearson, J. M. H., Rees, R. J. W. and Weddell, A. G. M. (1970). Mycobacterium leprae in the striated muscle of patients with leprosy. Lepr. Rev. 41, 155.

Pearson, J. M. H. and Weddell, A. G. M. (1971). Changes in sensory acuity following radial nerve biopsy in patients with leprosy. Brain 94, 43.

Pearson, J. M. H. and Weddell, A. G. M. (1975). Perineurial changes in untreated leprosy. Lepr. Rev. 46, 51.

Sabin, T. D., Hackett, E. R. and Brand, P. W. (1974). Temperatures along the course of certain nerves of ten affected in lepromatous leprosy. Int. J. Lepr. 42, 38.

Sheskin, J., Magora, A. and Sagher, F. (1969). Motor conduction velocity studies in patients with leprosy reaction treated with thalidomide and other drugs. Int. J. Lepr. 37, 359.

Ridley, D. S. (1969). Reactions in leprosy. Lepr. Rev. 40, 77. 